\title{
A Heat Loss Compensated Calorimeter and Related Theorems
}

\author{
Steve R. Domen \\ Center for Radiation Research, National Bureau of Standards, Washington, D.C. 20234 \\ (January 17, 1969)

\begin{abstract}
A new calorimetric design and measuring technique are proposed for reducing uncertainties caused by temperature gradients. A theoretical analysis reveals a mathematical theorem concerning heat transfer and its electrical analog.
\end{abstract}

Key words: Absorbed dose; calorimeter; heat loss compensation; heat flow theorems.

\section{Introduction}

In calorimetry of moderate accuracy it is sometimes considered sufficient to measure the temperature changes of the separate bodies comprising the calorimeter at only a single point in each, and, in treating the heat evolution and leakage during a run, to ignore temperature gradients of the bodies. The systematic errors produced by gradients that are not negligible have been discussed, [1,2] ${ }^{1}$ and upper limits for these errors have been assigned in some simple cases [3]. Experimental means are also available for detecting and evaluating the gradient-induced errors [4]. A calorimeter design is reported here which is intended essentially to eliminate systematic errors caused by temperature gradients. The instrument is intended for the measurement of the absorbed dose deposited in materials by beams of ionizing radiation, but the principles are applicable to calorimetry in general.

\section{Essential Elements}

The essential concept consists of a calorimetric body, the core, completely surrounded by a thermally isolated jacket of equal heat capacity. Figure 1 is a diagrammatic representation of a core and jacket of any possible shape. A thermistor is imbedded in each to indicate temperatures. The two thermistors form opposite arms of a Wheatstone bridge that is initially balanced when the core and jacket temperatures are at equilibrium. Unbalanced output voltages for small changes in temperature are, therefore, proportional

\footnotetext{
${ }^{1}$ Figures in brackets indicate the literature references at the end of this paper.
}

to the sum of the temperature changes of the core and jacket from equilibrium. The jacket is surrounded by a shield that is caused either to remain at a constant

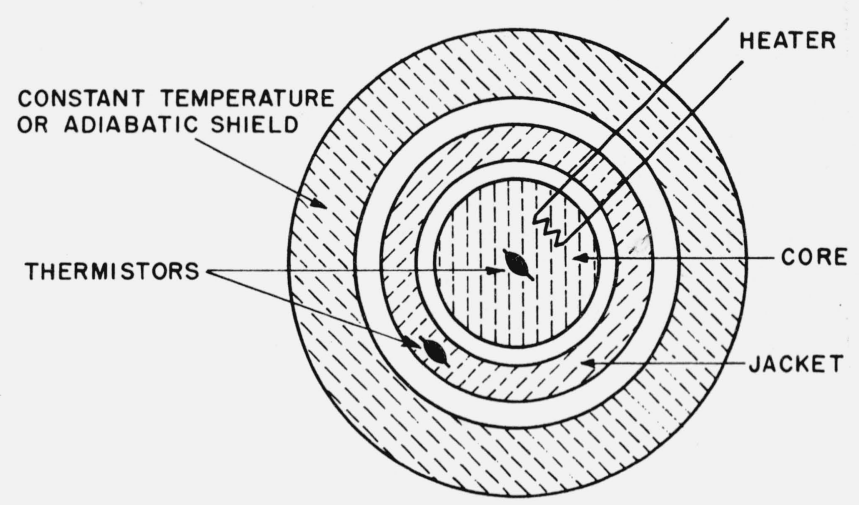

FIGURE 1. Essential elements of the heat loss compensated calorimeter.

temperature, or to follow the rising temperature of the jacket, depending upon the mode of operation that is chosen.

\section{Principle of Compensation}

Assume that power is to be applied to the core, first by an eccentrically located imbedded electric heater and then by a uniform absorption of energy produced from a radiation field. These two ways of applying power will produce large differences in temperature gradients throughout the core. If the gradients are large, as with an eccentrically located heater, the heat 
lost from the core during the application of power may be difficult to evaluate with satisfactory accuracy, especially if the core temperature is measured at only one point. However, the heat lost from the core that is retained in the jacket contributes to the output of the Wheatstone bridge just as if it had been retained in the core. ${ }^{2}$ Compensation for heat lost from the core by this method is nearly independent of the temperature gradients that are produced in the core during power application. Hence this method would seem to reduce the systematic error when the calorimeter is used to compare power sources that produce different temperature distributions in the core. The relatively small jacket leakage correction that remains can be assumed to be made with good accuracy, in the case where the shield is maintained at constant temperature, because the temperature rise of the jacket is smaller and more uniform than that of the core. For the same reasons, in the adiabatic mode, the temperature of the shield can be caused to follow the rising temperature of the jacket more readily and accurately than that of an unjacketed core.

\section{Application to Absorbed Dose Determination}

Now consider specifically the case of a calorimeter to be used for the measurement of absorbed dose in a radiation field. It is assumed that the shield remains fixed in temperature and that core and jacket are made of the same material and have equal heat capacities. The sensitivity of the calorimeter is determined from calibration runs in which measured power is supplied to and dissipated in the core by means of an imbedded electrical heater. No power is supplied to the jacket; its slight heating is the result of heat transferred to it from the core. The output voltage of the bridge circuit indicates $T_{1 C}+T_{2 C}$ where $T_{1 C}$ and $T_{2 C}$ are, respectively, the temperature changes of the core and the jacket from equilibrium, uncorrected for heat losses. Heat loss corrections can be determined by the usual methods of calorimetry.

When measurement runs are made the Wheatstone bridge is altered by substituting for the jacket's thermistor a fixed resistor of equal resistance external to the calorimeter. In this case the output voltage of the bridge indicates $T_{1 M}$, the temperature change from equilibrium of the core. The energy, $E_{M}$, absorbed by the core from the radiation is computed to be

$$
E_{M}=E_{C} \times T_{1 M}^{\prime} /\left(T_{1 C}+T_{2 C}\right)^{\prime},
$$

where $E_{C}$ is the measured electrical energy, supplied during the calibration, and $T_{1 M}^{\prime}$ and $\left(T_{1 C}+T_{2 C}\right)$ are the corrected temperature changes in the two runs.

${ }^{2}$ A somewhat different method is described in [5]. Calorimeters using jackets that are not a part of the heat measuring system are described in [6].

\section{Heat Transfer Relation}

It was discovered, during calculations of the ideal performance of the proposed single jacketed calorimeter design, that $T_{1 M}$ is identical to $T_{1 C}+T_{2 C}$, provided (1) that the absorbed radiation power converted to heat in the core and jacket are each the same as the electrical power converted to heat in the core during a calibration run, (2) that temperature changes are small enough so that heat capacities and heat transfer coefficients can be considered constant, and (3) that there are no temperature gradients within the core or jacket.

More generally, for a core surrounded by $N-1$ thermally isolated jackets,

$$
T_{1 M} \equiv T_{1 C}+\alpha_{2} T_{2 C}+\alpha_{3} T_{3 C}+\ldots .+\alpha_{N} T_{N C}
$$

if the same schedule of power, $P(t)$, is applied to the core during a calibration run and a measurement run, and if $\alpha_{2} P(t), \alpha_{3} P(t), \ldots ., \alpha_{N} P(t)$ are the schedules of power applied during a measurement run to the first jacket, the second jacket, etc., respectively, where $\alpha_{2}, \alpha_{3}, \ldots, \alpha_{N}$ are constants. This expression is independent of the possibly different heat capacities of the core and jackets, and also independent of the time variation of $P(t)$, whether $P(t)$ acts as a heater or as a refrigerator. A proof is given here for the case of a core and one jacket.

Let $C_{1}=$ the heat capacity of the core;

$C_{2}=$ the heat capacity of the jacket;

$T_{1}=$ the temperature change from equilibrium of the core for any run;

$T_{2}=$ the temperature change from equilibrium of the jacket for any run;

$K_{1}=$ the heat transfer coefficient between the core and jacket;

$K_{2}=$ the heat transfer coefficient between the jacket and the constant temperature shield;

$F=1$ for measurement runs; and

$F=0$ for calibration runs.

The temperatures are governed by the following differential equations of heat flow:

and

$$
C_{1} d T_{1}=P(t) d t-K_{1}\left(T_{1}-T_{2}\right) d t
$$

$$
C_{2} d T_{2}=F \alpha_{2} P(t) d t+K_{1}\left(T_{1}-T_{2}\right) d t-K_{2} T_{2} d t .
$$

Rearranging terms and substituting $D$ for the operator $d / d t$, the following forms are obtained:

$$
\left[D+\frac{K_{1}}{C_{1}}\right] T_{1} \quad-\frac{K_{1}}{C_{1}} T_{2}=\frac{P(t)}{C_{1}}
$$

and

$$
-\frac{K_{1}}{C_{2}} T_{1}+\left[D+\frac{1}{C_{2}}\left(K_{1}+K_{2}\right)\right] T_{2}=\frac{F \alpha_{2} P(t)}{C_{2}} .
$$


Solving for the temperature rise, $T_{1 M}$, produced in a measurement run in determinant form:

$$
T_{1 M}=\left|\begin{array}{cc}
\frac{P(t)}{C_{1}} & -\frac{K_{1}}{C_{1}} \\
\frac{\alpha_{2} P(t)}{C_{2}} & {\left[D+\frac{1}{C_{2}}\left(K_{1}+K_{2}\right)\right]}
\end{array}\right|
$$

$\frac{\left[D+\frac{1}{C_{2}}\left(K_{1}+K_{2}\right)+\frac{\alpha_{2} K_{1}}{C_{2}}\right] \frac{P(t)}{C_{1}}}{\Delta}$.

Similarly, the temperature rises, $T_{1 C}$ and $T_{2 C}$ produced during a calibration run are

$$
T_{1 C}=\frac{\left[D+\frac{1}{C_{2}}\left(K_{1}+K_{2}\right)\right] \frac{P(t)}{C_{1}}}{\Delta}
$$

and

$$
T_{2 C}=\frac{\frac{P(t) K_{1}}{C_{1} C_{2}}}{\Delta} .
$$

Thus

Hence

$$
T_{1 C}+\alpha_{2} T_{2 C}=\frac{\left[D+\frac{1}{C_{2}}\left(K_{1}+K_{2}\right)+\frac{\alpha_{2} K_{1}}{C_{2}}\right] \frac{P(t)}{C_{1}} .}{\Delta}
$$

$$
T_{1 M} \equiv T_{1 C}+\alpha_{2} T_{2 C} \text {. }
$$

It may be of interest to note the electric analog which is immediately obvious from the heat problem solved here. The circuits are shown in figure 2 . If the currents

produced by the generators are $\dot{\mathrm{l}}_{2}=\alpha_{2} \dot{\mathrm{l}}_{1}, \mathrm{i}_{3}=\alpha_{3} \mathrm{i}_{1}$, etc., then, regardless of how the ganged switches are operated,

$$
V_{1 M} \equiv V_{1 C}+\alpha_{2} V_{2 C}+\alpha_{3} V_{3 C}+\ldots .+\alpha_{N} V_{N C} .
$$

This is a special case of Lorentz' reciprocity relationship derived from Maxwell's equations [7]. The heat flow theorem, $T_{1 M} \equiv T_{1 C}+\alpha_{2} T_{2 C}$, has a useful application to the measurement of absorbed dose with the heat loss compensated calorimeter. It means that when a calibration run and a measurement run with $\alpha_{2}=1$ are of equal duration, the powers applied to the core are in the ratio of the $\mathbf{W}$ heatstone bridge output signals uncorrected for heat losses. This special case where $\alpha_{2}=1$ was considered in order to predict the temperature-time variations of the core and jacket. The special case does not have to be duplicated nor the above identity used for calorimetric energy determinations, but then heat loss corrections must be made by the usual methods.

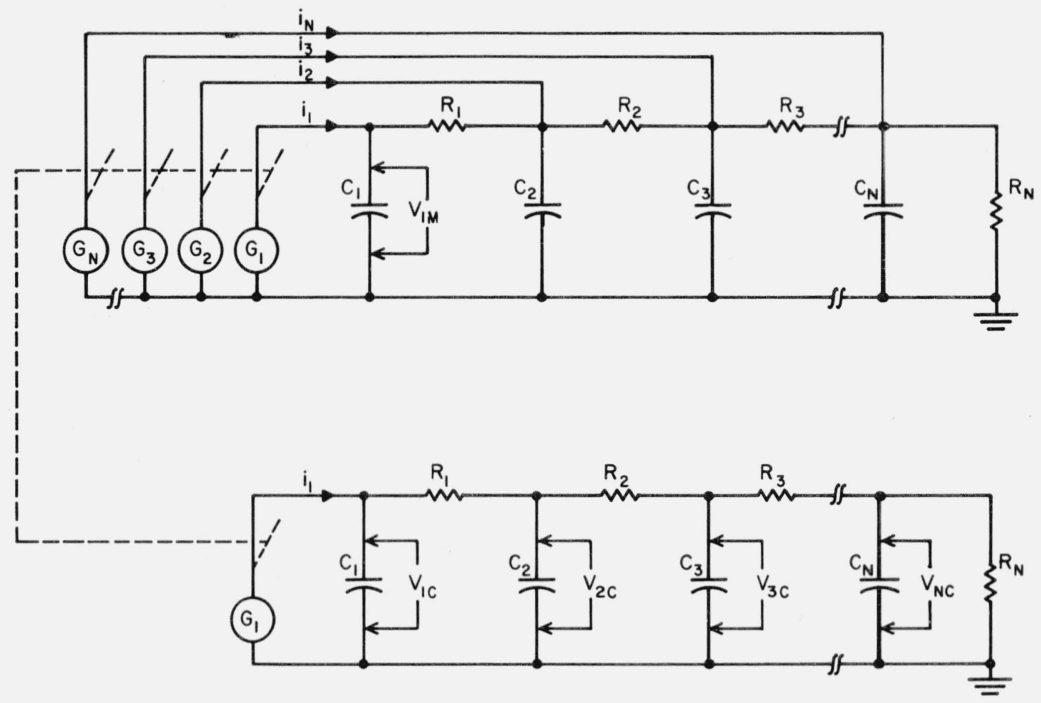

FIGURE 2. Equivalent electrical circuits resulting from the studies of the heat loss - . compensated calorimeter. If $\mathrm{i}_{2}=\alpha_{2} \mathrm{i}_{1}, \mathrm{i}_{3}=\alpha_{3} \mathrm{i}_{1}, \ldots \alpha_{N} \mathrm{l}_{1}$, then $V_{1 M} \equiv V_{1 C}+\alpha_{2} V_{2 C}+\alpha_{3} V_{3 C}+\ldots+\alpha_{N} V_{N C}$ 
The author is indebted to Ben Petree for his helpful comments. This work was supported by the National Cancer Institute, National Institutes of Health, Bethesda, Maryland, and by the Division of Biology and Medicine of the United States Atomic Eenergy Commission, Washington, D.C. A calorimeter of the design described for measuring absorbed dose is nearing completion in the NBS Center for Radiation Research, and an experimental program is under way.

\section{References}

[1] West, E. D., Heat exchange in adiabatic calorimeters, J. Res. NBS $67 \mathrm{~A}$ (Phys. and Chem.) No. 4, 331-341 (1963).

[2] Laughlin, J. S., and Genna, S., Calorimetry in Radiation Dosimetry, edited by F. H. Attix and W. C. Roesch, Vol. II, 421
(Academic Press, New York, 1966).

[3] Petree, B., and Ward, G., The construction of calorimeters for the measurement of absorbed dose, NBS Tech. Note 163 (1962).

[4] Petree, B., and Lamperti, P., A comparison of absorbed dose determination in graphite by cavity ionization measurements and calorimetry, J. Res. NBS 71C (Engr. and Instr.) No. 1, 19 (1967).

[5] Persoz, B., Nouvelles methods de measure de la chaleur specifique vraie des metaux a haute temperature, Ann. Phys. (Paris 15, 327 (1940).

[6] McCullough, J. P., and Scott, D. W., editors, Experimental Thermodynamics, Vol. I, (Plenum Press, New York, 1968).

[7] Carlin. H. I.. and Giordano, A. B., Network Theory: An Introduction to Reciprocal and Nonreciprocal Circuits, p. 350 (Prentice-Hall, Inc., Englewood Cliffs, New Jersey, 1964).

(Paper 73Cl \& 2-286) 